\title{
Construcción de estrategias metodológicas para la mejora de la práctica docente, en cuatro escuelas públicas del barrio Acahualinca, Managua-Nicaragua
}

\author{
Isabel Cristina Lazo Montenegro ${ }^{1}$ \\ Ertilia Herrera Herrera ${ }^{2}$
}

\section{RESUMEN}

La Investigación realizada, es la experiencia compartida por 8 compañeras docentes de las escuelas públicas de Acahualinca, que han realizado la reflexión de su práctica docente, la construcción colectiva y colaborativa de las docentes que conllevó a la implementación de estrategias metodológicas en el aula de clase, con niños y niñas de Educación Inicial y Primaria. El propósito es mejorar la calidad de la educación y la práctica docente, a través de reflexionar su quehacer docente, tomando en cuenta el contexto, su práctica y la experiencia vivida desde el aula de clase y fuera de ella. La investigación se desarrolló en el período comprendido de marzo del 2013 hasta agosto del 2014. Se trabajaron los siguientes procesos: formación, elaboración diagnóstico, acción, evaluación de la Investigación-acción. Los resultados son muy significativos, ya que las docentes lograron aplicar las diferentes estrategias metodológicas y sobre todo lograron tomar conciencia sobre la importancia de reflexionar sobre su práctica, ya que a través de este proceso lograron contagiar a sus estudiantes y comunidad educativa sobre la importancia de construir colectivamente y aprender desde la experiencia y cambio de actitud frente a los aprendizajes.

Palabras claves: reflexión de la práctica, aprendizajes, calidad, estrategias, construcción.

Recibido: 10 de diciembre de 2014

Aceptado: 10 de marzo de 2015

1 Maestra en Gestión del Desarrollo Comunitario. Docente de Educación Media con mención en Ciencias Sociales. Posgrado en didáctica de la CCSS, en Historia de Nicaragua. Coordinadora de atención a centros públicos a nivel nacional de Fe y Alegría Nicaragua. Correo Electrónico: josae12003@yahoo.es

2 Master en Planificación ambiental y gestión de recursos naturales, especialista en gestión del desarrollo comunitario y en docencia universitaria. Correo Electronico: eherrerah06@yahoo.es 


\title{
Construction of methodological strategies for the improvement of the teaching practice in four public schools in the district Acahualinca, Managua-Nicaragua
}

\begin{abstract}
The research conducted is the shared experience of 8 teachers from public schools in Acahualinca, who have made the reflection of their teaching practice, the collective and collaborative construction of teaching that led to the implementation of methodological strategies in the classroom with children in Early and Primary Education. The purpose is to improve the quality of education and the teaching practice through reflection teaching work, taking into account the context, the practice, and the experience from the classroom and beyond. The research was conducted in the period March 2013 to August 2014. The following processes were worked: formation, diagnosis, preparation, action, evaluation of the Action Research. The results are very significant since the teachers were able to apply the different methodological strategies, and mostly managed to take awareness of the importance of reflecting on their practice since through this process they managed to infect their students and school community with the importance of building collectively and learning from experience and changing the attitude towards learning.
\end{abstract}

Keywords: reflection of practice, learning, quality, strategies, construction. 


\section{INTRODUCCIÓN}

La reflexión de la práctica docente, es uno de los cuestionamientos o planteamientos que se han venido promoviendo en los a lo largo de varias décadas, como una forma de romper paradigmas y construir nuevos conocimientos desde el aula de clase, donde el docente, los estudiantes y el apoyo de la comunidad educativa sean los principales protagonistas de transformar una educación de calidad, integral, para la vida y por consiguiente la transformación de la realidad, tanto personal como de la comunidad.

La investigación Acción trabajada con las 8 docentes de las escuelas de Acahualinca, Managua, Nicaragua, tiene como objetivo la construcción de estrategias metodológicas para de promover en las docentes de cuatro escuelas públicas de Acahualinca la mejora de la práctica desde un enfoque de Educación Popular, para aportar a la transformación de las realidades de los y las sujetos en el aula de clase, importancia que esto tiene para el cambio de una educación de calidad, con la participación de todas y todos, con nuevas metodologías que ayudan a transformar las realidades de los y las docentes; así como las realidades de los y las estudiantes y de la comunidad.

La reflexión del trabajo docente sirvió para detenerse a pensar, meditar y cuestionarse sobre la importancia de construir colectivamente como equipo docente y con los y las estudiantes; así como el apoyo de los padres y madres de la comunidad involucrada en el proceso.

Reflexionar la práctica desde el enfoque de la educación popular, no es solo quedarnos en la acción cotidiana desde el aula de clase, significa analizar la práctica desde una triple mirada: concepciones que tengo desde mi práctica, el contexto en el que la llevo a cabo, la práctica concreta que realiza (Núñez).

Compartir la experiencia vivida en la realización de la Investigación-Acción-Participativa es con el objetivo de motivar y cuestionar a la reflexión de la práctica desde el aula de clase, donde cada docente puede autoevaluarse para recrear su aprendizaje. Es por eso que cada una de las docentes participantes en este proceso aporta estrategias novedosas desde la reflexión de su práctica como docentes, desde el aula y la comunidad.

Esta investigación se realizó bajo en paradigma socio crítico. El paradigma tiene sus antecedentes en la Escuela de Frankfurt, en Carr y Kemnis, tiene como objetivo el análisis de las transformaciones sociales.

El enfoque es investigación acción participativa (IAP) la cual han venido construyendo diferentes autores como Kurt Lewin, Stenhouse, John Elliott, Kemmis, McTaggart entre otros.

\section{MATERIALES Y MÉTODOS}

Cabe mencionar que este trabajo de investigación se realizó en dos dimensiones y dos grandes momentos:

En una primera etapa o momento se realizó el acompañamiento desde Fe y Alegría, a través de la facilitadora y autora de esta investigación generando procesos de reflexión desde el enfoque de la Educación Popular; para generar procesos de transformación y cambio social en la persona, la escuela y la comunidad. En ese momento se realizó El triple autodiagnóstico, "es un método que parte del conocimiento general de los distintos elementos que están presentes en la realidad y en la concepción de la comunidad" (Caal, Rubén Antonio, 29 de abril 2011).

Carlos Núñez, educador popular mexicano nos facilita un mejor entendimiento del triple autodiagnóstico, visualiza los tres terminos o aspectos de triple autodiagnóstico; Contexto, concepción y práctica. Según el autor, "Desarrollar un proceso de autodiagnóstico participativo requiere considerar la compleja relación personal y social entre los hechos objetivos y las interpretaciones subjetivas que de los 
mismos hechos tienen los individuos y los grupos". La herramienta concreta de análisis consiste en la realización del proceso; reconocimiento de la "concepción" que sobre el tema y/o realidad se tenga del "contexto" donde se actúa y de "la práctica misma" que se realiza. En esta investigación podemos decir que es autodiagnóstico porque cada una/uno de forma individual; así como de forma colectiva se analizaron y evaluaron su práctica, se autoevaluaron y de ellas/ ellos surgen los planteamientos a trabajar tomando en cuenta el contexto, la práctica y los conocimientos teoricos conceptuales de cada una/uno de los participantes.

Esta primera etapa de la investigación se realizó en 2013, participaron 45 docentes de los centros educativos, cinco docentes por centro, 43 mujeres 3 varones. Se procedió a un proceso de formación para generar el autodiagnóstico y la reflexión de la práctica en el aula de clase. El proceso diagnóstico se realizó a través de 6 talleres de grupos focales, las sesiones se realizaron cada quince días, se realizó la reflexión del contexto, tomando en cuenta los siguientes ámbitos: económicos, sociales, culturales. Bajo la lógica ¿Dónde estoy?, ¿cómo está mi centro?. También se aplicó una entrevista a 10 de los docentes que participaron el proceso de formación, seleccionados al azar, con el objetivo de conocer su opinión de sobre los talleres de estrategias metodológicas, a través de la reflexión de la práctica docente; esto fortaleció el trabajo del diagnóstico y la identificación del problema a trabajar por centros.

Los docentes identificaron su problemática, a través de una construcción colectiva por centro y por colectivo participante de los talleres. Las reflexiones, cuestionamientos y contradicciones fueron significativos; ya que se analizó y discutió los aspectos de contexto y problemática encontradas en cada centro. Se desarrolló un segundo momento el cuál consistió en la construcción de estrategias metodológicas para mejorar su quehacer docente en el aula de clase, análisis de contenido y la reflexión crítica para mejorar la calidad educativa y generar proceso de aprendizajes significativos.

En la segunda etapa realizada hasta agosto 2014 participaron en el proceso 8 docentes mujeres, quienes atienden a los niños-niñas, adolescentes de educación inicial, primaria regular y extra edad de los turnos matutino de los centros participantes. Constituyendo 5 casos que consistieron en:

- Caso 1: una docente que atiende preescolar, escuela Oasis de Esperanza y que trató el tema de familia.

- Caso 2: dos docentes en la escuela Oasis de Esperanza y trato el tema, estrategias de enseñar a leer para mejorar la disciplina.

- Caso 3: dos docentes de la escuela Anexo Juan Pablo II, trató el tema, disciplina en el aula de clase

- Caso 4: dos docentes de la escuela Wisconsin, tema, estrategias de comprensión lectora.

- Caso 5: una docente de la escuela Cristo Obrero, trató el tema de criterios de evaluación.

El proceso se inició con el análisis y discusión de los equipos directivos que deciden participar junto con sus docentes, como una forma de dar salida a las necesidades encontrada en el colectivo docente y una alternativa de transforma el aprendizaje para transformar realidades.

Se retomó los resultados del triple autodiagnóstico donde definieron el problema a reflexionar, de acuerdo a las necesidades encontradas por las diferentes escuelas.

Se utilizó la técnica de grupos focales para compartir las experiencias de la reflexión de la práctica, la construcción de los planes de acción. En conjunto con las docentes elaboramos un plan de acción, dónde se refleja las actividades a seguir para mejorar la práctica actual. En él se plasma la ruta de acompañamiento y seguimiento para observar la acción y recoger las evidencias que permitirán evaluar la aplicación de las 
diferentes estrategias metodológicas implementadas. Los acompañamientos y seguimiento a la implementación de las estrategias fueron programadas en conjunto con cada una de las docentes participantes de la Investigación; así como el registro de la información.

\section{RESULTADOS}

Los y las docentes compartieron resultados de las sesiones de los talleres y llegaron alas siguientes conclusiones por aspectos:

Debemos de bridar mas atención, centrar la atención en los niños y la niñas.

Reflexionar que lo que estoy dando es lo suficiente. Cómo lo hago, según tiempo y espacio.

Debemos de ser más observadores y humanista. Cuestionarnos sobre la actitud que debemos de tener frente al estudiante.

Se propone planificar tomando en cuenta el contexto y mediante estrategias de aula, para facilitar en los y las estudiantes la motivación, su autonomía en los procesos de aprendizaje, herramientas para que puedan leer bien, comprender bien, resolver problemas y saberlos aplicar en la vida cotidiana. En todo el proceso se retomara el tema de violencia y género.

Manejar las concepciones desde la práctica y la teoría desde el enfoque de Educación Popular. ¿Para qué?, para ver cómo vamos transformando desde nuestra práctica nuestras ideas y concepciones. Un paso importante fue la confrontación de nuestra práctica, con el contexto y nuestra concepción. Nos dimos cuenta de las contradicciones y coherencias en nuestra práctica. Esto ayudó a definir las prioridades sobre estrategias de intervención.
Otros aspectos significativos, fueron los resultados de la encuesta aplicada a un grupo de docentes participantes de los grupos focales.

E1 70\% de los docentes encuestados está consciente de lo que significa ser educador popular, sin embargo el $30 \%$ todavía no está sensibilizado de esta importancia E1 70\% de los docentes considera que maneja el término de educación popular y la importancia que tiene esta para lograr cambios en las injusticias sociales; sin embargo el $30 \%$ no es consciente de la importancia del término y del significado de la educación popular.

EL 70\% coincide que para efectuar su práctica docente toma en cuenta el contexto, el $30 \%$ considera que tiene que trabajar más en este ámbito, ya que es indispensable en el aprendizaje de los niños y niñas.

Los docentes toman en cuenta los conocimientos previos de los y las estudiantes, el $30 \%$ considera que no los toman en cuenta.

Las docentes decidieron retomar estrategias para mejorar la comprensión lectora y la disciplina en el aula de clase (etapa de acción).

\section{Resultados del desarrollo de las estrategias metodológicas}

Caso $N^{\circ}$ 1: Definición del concepto de Familia, trabajada por la profesora Silvia Ocon con los niños y niñas de la edad de pre-escolar. Colegio Cristiano Oasis de Esperanza

En el centro Oasis de Esperanza, la docente Silvia Ocon, trabajó con los y las niñas en edad pre-escolar el tema de la conceptualización de la familia.

He analizado el contexto familiar de cada uno de los niños encontrando diferentes situaciones que infieren en cada grupo familiar, donde ha habido separaciones, abandono, $\mathrm{y}$ diversos problemas que el niño de una $\mathrm{u}$ otra manera se ven afectado. 
De acuerdo a esta problemática, mi práctica como docente en relación al tema de la Familia, contenido que se tiene que impartir según el MINED en los ámbitos de educación en educación inicial. Al impartir este contenido me he formulado ciertas interrogantes que me han ayudado a reflexionar, y crear conciencia del concepto de familia en las niñas y niños.

¿Qué estrategias he utilizado? En primer lugar trabajo un ambiente en el aula de clase para que el niño y la niña reconozcan su familia y se identifique dentro de su núcleo familiar.

Elaboré los rincones de aprendizajes, en el trabajo de pre-escritura y pre-lectura, donde retomé las láminas de la familia.

Realicé en conjunto con la familia "un día de verano

Caso $N^{\circ}$ 2: Construcción de estrategias metodológicas de enseñanza de lectura en primer grado, como un mecanismo para mejorar la disciplina en el aula de clase. Las docentes Diana y Karolina de Oasis de Esperanza.

\section{Las docentes nos cuentan el proceso seguido: Defino el problema:}

El problema que tenemos con nuestros niños/as es la Disciplina dentro del aula de clases. Trabajamos con niños/as que viven en hogares disfuncionales, padres sumergidos en el ambiente de la drogadicción, prostitución, robo y violencia intrafamiliar. Esto nos ha afectado en gran manera, pues la forma de reflejar la problemática vivida en el hogar es la agresividad como un mecanismo de defensa ante las desigualdades que existen entre ellos mismos como estudiantes, problemas familiares, y la violencia que reciben antes de entrar al salón de clases (padres agrediendo física y verbalmente al niño/a en la calle).

\section{Preguntas de reflexión para la acción:}

Debido a las muchas incidencias disciplinarias presentadas en nuestros niños/as, han surgido diversas preguntas en cuanto a nuestro trabajo como Docente.

\section{¿QUÉ hago? ¿Cómo?}

Al hacer conciencia de la problemática presentada en nuestro salón de clases, iniciamos a documentarnos sobre estrategias que podíamos utilizar para mejorar la disciplina de nuestros estudiantes, aplicando, modificando y adecuando a nuestro contexto cada una.

\section{¿Cuándo?}

Durante el desarrollo de la jornada laboral realizamos la aplicación de nuestras estrategias didácticas, en pro de mejorar la disciplina en cada niño/a a nuestro cargo.

\section{¿En que necesito mejorar?}

Investigar constantemente sobre estrategias que estén vinculadas a nuestro tema.

Brindar una atención individualizada con aquellos niños que están en constante cambio disciplinario, inquietándonos a conocer la problemática con el fin de incidir desde mi posición como Docente.

Acontinuación describimos las estrategias desarrolladas con niños y niñas para mejorar la disciplina y agilizar la fluidez lectora:

- Lectura imaginativa (dibujan) logre notar en los niños que les llama más la atención ir dibujando según su imaginación relacionándolo con lo escuchado, ilustrar el cuento, esto provocó que la lectura despertara interés, atención en el estudiante a través del dibujo.

- Estrategia el semáforo de comportamiento, ha servido para reflexionar sobre cómo trabajar en el aula de clase; nos avisa como estamos en la clase, bien-seguimos la clase, mal nos alertamos para mejorar la disciplina, muy mal nos detenemos, 
no continuamos y conversamos de forma grupal y reflexiva para mejorar en caso poseen un color.

- Mi pizarrita. Esta herramienta es elaborada de forma artesanal por los niños y niñas con apoyo de los padres y madres. A través de esta estrategia el niño mejora su comportamiento puesto que se motiva al mirar a otro lograrlo y si él presenta dificultad se interesa en sí mismo por mejorar. Se hace uso de la autoevaluación

\section{Caso 3: Investigación de campo para la construcción} de Mitos y Leyendas en la comunidad, como una estrategia metodológica para mejorar la disciplina en el aula de clase de 5 to y 6 to grado de primaria del centro educativo Juan Pablo II. Las docentes Adriana Quintanilla, Irelia

\section{Pregunta de investigación:}

¿Cómo mejorar la disciplina en los estudiantes de de 5 to y 6 to grado de primaria?

\section{Conceptualizar y contextualizar sobre disciplina:}

¿Qué negociar con las y los estudiantes?

A partir de la reflexión se definieron las estrategias a utilizar que se describen a continuación y que fueron construidas colectivamente: Construcción de un libro de mitos y leyendas de la comunidad del barrio los Martínez - Pantanal, con el fin de desarrollar competencias de localización e investigación por parte de los estudiantes en conjunto con miembros de la comunidad.

El libro fue elaborado por los y las niños de una de las escuelas involucradas en el proceso investigaciónacción, con el apoyo de la comunidad de los Martínez - Pantanal, líderes de la comunidad, padres de familia, docentes. El libro narra las apariciones de la mona, el hombre del pantanal y a la mujer de blanco entre otras. Los estudiantes han utilizado, herramientas de las TIC, laptop computadora XO para tomar videos, fotos así mismo celulares, computadora portátil para la obtención de la información y redacción del libro de mitos y leyendas.

Los estudiantes del 5to y 6to han participado en la elaboración de foros, donde ellos compartieron sus vivencias, la Idiosincrasia de los pueblos. En estos foros participaron los padres de familia y líderes de la comunidad, los estudiantes se van afianzando en valores, apropiándose de su rol como estudiante activoparticipativo, creativo.

Caso 4: Comprensión lectora en los estudiantes de 5 to y 6 to grado mediados por las TICs (tecnología de la información y la comunicación) centro educativo Wisconsin. Las docentes Maritza Téllez, Sonia y Lesbia

Los estudiantes de sexto y quinto grado del turno matutino, han logrado motivación, integración y mejora de la comprensión lectora, a través la combinación de las TIC, en el proceso de aprendizajes.

Con la integración de las Tics a las actividades pedagógicas se ha logrado: La motivación y el interés por comprender la lectura de textos lo cual ha contribuido al logro y a la efectividad del proceso de aprendizaje. Con esta iniciativa el estudiante ha desarrolla la imaginación creadora a través de la dramatización, expresión oral, análisis y síntesis, incorpora nuevas palabras enriqueciendo su vocabulario lo que permite desarrollar su expresión escrita.

Motiva el interés investigativo por conocer diferentes culturas a través de la lectura; por consiguiente la disciplina, la cooperación, la solidaridad y el trabajo en equipo.

Caso 5: Proyectos de aula. Estrategias de evaluación con estudiantes de 6to grado de primaria, Centro educativo Cristo Obrero.

\section{Docente Yolanda}




\section{Estrategia de evaluación desarrollada:}

En relación a las estrategias de evaluación, se realizó una construcción colectiva con los estudiantes, ellos/ellas fueron participes y tomados en cuenta en la elaboración de las estrategias y los criterios de evaluación. Decidieron la forma de evaluarse, la calificación, las fechas y las estrategias a trabajar para mejorar en el aprendizaje.

Se construyeron y aplicaron diferentes estrategias como: exposiciones creativas, dramatizaciones, conversatorios sobre la dificultad del trabajo de las palabras agudas, proyecto de aula y recital de poesía.

Una de las estrategias muy significativa, fue el proyecto de aula; ya que se trabaja de forma integral y transdisciplinar. Para cada una de las estrategias antes mencionadas, se construyeron los siguientes criterios: creatividad, redacción, expresividad, soltura, trabajo en equipo, elaboración de dibujos, entre otros. Los y las estudiantes fueron conscientes de cada uno de los criterios elaborados para evaluar.

\section{CONCLUSIONES}

La construcción de estrategias metodológicas para lograr un mejor aprendizaje y cambio de conductas y actitudes en los estudiantes debe partir de conocer el contexto donde viven los estudiantes, sus problemas, intereses, sueños y esperanzas; y por otro lado debe partir de la reflexión crítica de cada docente donde cuestione su práctica y tome decisiones para logar un cambio en primer lugar de sí mismo.

Es importante destacar que cada uno de los momentos trabajados en la reflexión de la práctica ha ayudado y cuestionado a las docentes en la forma de compartir el aprendizaje; así como el involucramiento de los equipos directivos y comunidad educativa en general.

Este proceso ayudo a comprender la forma de aprendizaje de parte de las docentes que reflexionaron la práctica como de la facilitadora que apoyo el proceso de formación y acompañamiento.

\section{RECOMENDACIONES}

Las docentes recomendaron los siguientes aspectos que se mencionan a continuación.

- La adecuación curricular y las estrategias de aula desde educación popular.

- Planificación e implementación de un enfoque curricular, integral, transversal, inclusivo y transformador desde la educación Popular.

- Continuar fortaleciendo la reflexión de la práctica docente a través de la Investigación-Acción

- Compartir con los y las docentes; así como con la comunidad educativa, los avances significativos de la experiencia.

- Registrar la información que se vaya trabajando desde el aula de clase, sobre todo registrar en el diario del investigador la expresiones y sentimientos de los y las estudiantes con las que trabajan.

- Contrastar la teoría con la práctica cotidiana y construir nuevos conocimientos desde la experiencia vivida desde la reflexión.

\section{BIBLIOGRAFÍA}

Alegría, F. y. (2012). Formación a Educadores. Bogotá: Fe y Alegría.

Alegría, F. y., \& Fe y Alegría. (2005). Pedagogia de la Educación Popular. Bogotá: Fe y Alegría.

Mejía, R. (2000). Ponencia presentado al Congreso de Fe y Alegía. Colombia: Fe y Alegría.

La Concepción Metodológica

Dialéctica, los Métodos y las Técnicas Participativas en la Educación Popular Oscar Jara Holliday

Educación Popular; Pedagogía de la Educación Popular; Educación crítica

Educar para transformar, transformar para educarCarlos Núñez Selección de textos

Van de Velde, Herman ... (ed.) Educación popular. Herman Van de Velde. Centro de Investigación, Capacitación y Acción Pedagógica (CICAP)/ Facultad Regional Multidisciplinaria (FAREM).$1^{\mathrm{a}}$. Ed. Estelí: 